\title{
A CHARACTERIZATION OF HOLOMORPHIC SEMIGROUPS
}

\author{
TOSIO KATO ${ }^{1}$
}

\begin{abstract}
A necessary and sufficient condition is given for a one-parameter semigroup $\{U(t)\}, 0 \leqq t<\infty$, of class $C_{0}$ on a Banach space to be holomorphic (of class $H\left(\Phi_{1}, \Phi_{2}\right)$ for some $\left.\Phi_{1}<0<\Phi_{2}\right)$. The condition is expressed in terms of the spectral properties of $U(t)-\zeta$ for small $t>0$ and for a complex number $\zeta$ with $|\zeta| \geqq 1$.
\end{abstract}

Let $\{U(t)\}_{0<t<\infty}$ be a semigroup of linear operators of class $C_{0}$ on a (complex) Banach space $X$ (see Hille-Phillips [1, p. 321]). In this note we give a necessary and sufficient condition for $\{U(t)\}$ to be a holomorphic semigroup, by which we mean a semigroup belonging to the class $H\left(\Phi_{1}, \Phi_{2}\right)$ for some $\Phi_{1}<0<\Phi_{2}[1$, p. 325]. Roughly speaking, we prove that $\{U(t)\}$ is holomorphic if and only if there is a point on the unit circle $|\zeta|=1$, different from 1 , that belongs to the resolvent set $\rho(U(t))$ of $U(t)$ for sufficiently small $t$.

In this connection it will be noted that for any $\{U(t)\}$ of class $C_{0}$, each complex number $\zeta$ with $|\zeta|>1$ belongs to $\rho(U(t))$ for sufficiently small $t$. In other words, the spectral radius $r(U(t))$ of $U(t)$ satisfies $\lim \sup _{t \rightarrow 0} r(U(t)) \leqq 1$. This follows immediately from the well-known inequality

$$
\|U(t)\| \leqq M e^{\beta t}, \quad 0<t<\infty,
$$

where $M$ and $\beta$ are constants.

Our result generalizes a recent result of Neuberger [2] to the effect that if $\lim \sup _{t \rightarrow 0}\|1-U(t)\|<2$ then $A U(t) \in B(X)$ for $t>0$, where $A$ denotes the infinitesimal generator of $\{U(t)\}$.

Precisely, our result is given by

Theorem. Let $\{U(t)\}$ be a semigroup of class $C_{0}$. The following three conditions are equivalent.

(i) $\{U(t)\}$ is a holomorphic semigroup.

(ii) For each complex number $\zeta$ with $|\zeta| \geqq 1, \zeta \neq 1$, there are $\delta, K>0$ such that

$$
\zeta \in \rho(U(t)), \quad\left\|(\zeta-U(t))^{-1}\right\| \leqq K \quad \text { for } 0<t<\delta .
$$

Received by the editors November 24, 1969.

AMS Subject Classifications. Primary 4730, 4750.

Key Words and Phrases. Semigroup of class $C_{0}$, holomorphic semigroup, resolvent set, spectral radius, operator calculus, semi-Fredholm operator, index.

1 Supported by AFOSR Grant 68-1462. 
(iii) There are positive numbers $\delta, K$ and a complex number $\zeta$ with $|\zeta|=1$ such that

$$
\|(\zeta-U(t)) x\| \geqq\|x\| / K \quad \text { for } x \in X \text { and } 0<t<\delta .
$$

Corollary. If there are $\delta, \epsilon>0$ such that $\|1-U(t)\| \leqq 2-\epsilon$ for $0<t<\delta$, then $\{U(t)\}$ is holomorphic.

To deduce the corollary from the theorem, it suffices to note that (iii) is satisfied with $\zeta=-1$. In fact $\|(-1-U(t)) x\| \geqq 2\|x\|$ $-\|(1-U(t)) x\| \geqq \epsilon\|x\|$.

To prove the theorem, we use the infinitesimal generator $A$ of $U(t)$. Suppose $\{U(t)\}$ is holomorphic. Then $\rho(A)$ contains a sector $S=\{z \mid \arg (z-\beta)<\pi / 2+\omega\}$ with $\omega>0$, and

$$
\left\|(z-A)^{-1}\right\| \leqq M /|z-\beta|, \quad z \in S .
$$

$U(t)$ is given by

$$
U(t)=\frac{1}{2 \pi i} \int_{C} e^{t z}(z-A)^{-1} d z, \quad t>0,
$$

where $C$ is a curve running in $S$ from $\infty e^{-i \theta}$ to $\infty e^{i \theta}$ with $\pi / 2<\theta<\pi / 2$ $+\omega$. (These results are well known and may be found in [1], [3] or [4].)

Changing the variable of integration, we obtain

$$
U(t)=\frac{1}{2 \pi i} \int_{C} e^{z}(z-t A)^{-1} d z, \quad t>0,
$$

where we may assume that $C$ is independent of $t$ as long as $0<t<\delta$. Furthermore, the portion of $C$ in the right half-plane can be made as small as we like if $\delta$ is sufficiently small. In particular, given any $\zeta \neq 1$ with $|\zeta| \geqq 1$, we can achieve that $e^{z} \neq \zeta$ for all $z$ lying on $C$ or to the left of $C$.

With such a choice of $\delta$ and $C$, we define

$$
B(t)=\frac{1}{2 \pi i} \int_{C} e^{z}\left(e^{z}-\zeta\right)^{-1}(z-t A)^{-1} d z, \quad 0<t<\delta .
$$

The integral converges absolutely and defines an operator $B(t) \in B(X)$ (the set of all bounded linear operators on $X$ to $X$ ). Since (2) implies $\left\|(z-t A)^{-1}\right\| \leqq M /|z-t \beta| \leqq M^{\prime} /|z|$ for $z \in C$, we have

(6) $\|B(t)\| \leqq\left(M^{\prime} / 2 \pi\right) \int_{C}\left|z^{-1} e^{z}\left(e^{z}-\zeta\right)^{-1} d z\right|=M^{\prime \prime}, \quad 0<t<\delta$. 
Now we apply the Dunford-Taylor operational calculus to (4), (5), noting that the functions $e^{z}$ and $e^{z}\left(e^{z}-\zeta\right)^{-1}$ of $z$ are holomorphic on and to the left of $C$ and tend rapidly to zero as $\operatorname{Re} z \rightarrow-\infty$. Since the product of these two functions are equal to $e^{z}+\zeta e^{z}\left(e^{z}-\zeta\right)^{-1}$, we obtain $U(t) B(t)=B(t) U(t)=U(t)+\zeta B(t)$. Hence $\quad(\zeta-U(t))(1-B(t))=\zeta$ $=(1-B(t))(\zeta-U(t))$ and so

$$
(\zeta-U(t))^{-1}=\zeta^{-1}(1-B(t)), \quad\left\|(\zeta-U(t))^{-1}\right\| \leqq|\zeta|^{-1}\left(1+M^{\prime \prime}\right) .
$$

This proves that (i) implies (ii).

It is obvious that (ii) implies (iii).

To prove that (iii) implies (i), we use the identity

$$
e^{-i t \alpha} U(t) x-x=\int_{0}^{t} e^{-i s \alpha} U(s)(A-i \alpha) x d s, \quad x \in D(A),
$$

where $\alpha$ is any real number. Using (1), we thus obtain

$$
\begin{aligned}
\left\|\left(U(t)-e^{i t \alpha}\right) x\right\| & \leqq M \beta^{-1}\left(e^{\beta t}-1\right)\|(A-i \alpha) x\| \\
& \leqq M t(1-\beta t)^{-1}\|(A-i \alpha) x\|
\end{aligned}
$$

for $\beta t<1$.

Suppose (iii) is satisfied. Choose two positive numbers $\theta^{\prime}, \theta^{\prime \prime}$ such that $\zeta=e^{i \theta^{\prime}}=e^{-i \theta^{\prime \prime}}$. Let $\alpha>\max \left(\theta^{\prime} / \delta, \beta \theta^{\prime}\right)$ and set $t=\theta^{\prime} / \alpha$. Then $0<t<\delta$ and $\beta t<1$. Since $e^{i t \alpha}=e^{i \theta^{\prime}}=\zeta$, we have $\left\|\left(U(t)-e^{i t \alpha}\right) x\right\|$ $\geqq\|x\| / K$ by hypothesis. It follows from (7) that

$$
\begin{aligned}
\|(A-i \alpha) x\| & \geqq(1-\beta t)\|x\| / M K t \\
& =\left(\alpha-\beta \theta^{\prime}\right)\|x\| / M K \theta^{\prime}, \quad x \in D(A) .
\end{aligned}
$$

(8) implies that if $\alpha>\beta \theta^{\prime}, A-i \alpha$ is a semi-Fredholm operator with nullity 0 and minimum modulus $\geqq\left(\alpha-\beta \theta^{\prime}\right) / M K \theta^{\prime}$ (see $[4$, p. 230]). Hence $A-i \alpha-\xi$ is also semi-Fredholm if $|\xi|<\left(\alpha-\beta \theta^{\prime}\right) / M K \theta^{\prime}$ (see [4, p. 236]). But it is known that $i \alpha+\xi \in \rho(A)$ if $\xi>\beta$, so that ind $(A-i \alpha-\xi)=0$. Since the index is constant on any component of the semi-Fredholm domain (see [4, p. 243]), we conclude that $\operatorname{ind}(A-i \alpha)=0$ or, equivalently, $i \alpha \in \rho(A)$, provided $\alpha$ is sufficiently large. Then (8) implies that $\left\|(A-i \alpha)^{-1}\right\| \leqq M K \theta^{\prime} /\left(\alpha-\beta \theta^{\prime}\right)$ for sufficiently large $\alpha$.

Similarly one proves that $-i \alpha \in \rho(A)$ for sufficiently large $\alpha$, with $\left\|(A+i \alpha)^{-1}\right\| \leqq M K \theta^{\prime \prime} /\left(\alpha-\beta \theta^{\prime \prime}\right)$. As is well known (see [1], [3], or $[4])$, these inequalities show that $\{U(t)\}$ is holomorphic. Thus (iii) implies (i). 


\section{BibliograPHY}

1. E. Hille and R. S. Phillips, Functional analysis and semi-groups, rev. ed., Amer. Math. Soc. Colloq. Publ., vol. 31, Amer. Math. Soc., Providence, R.I., 1957. MR 19, 664.

2. J. W. Neuberger Analyticity and quasi-analyticity for one-parameter semi-groups, (to appear).

3. K. Yosida, On the differentiability of semi-groups of linear operators, Proc. Japan Acad. 34 (1958), 337-340. MR 20 \#5435.

4. T. Kato, Perturbation theory for linear operators, Die Grundlehren der math. Wissenschaften, Band 132, Springer-Verlag, Berlin and New York, 1966. MR 34 \#3324.

University of California, Berkeley, California 94720 